lement. L'activité constitutive du récepteur mGlula, qui résulte de la synthèse de Homer la, peut donc être considérée comme une mémoire cellulaire d'une hyperactivité transitoire antérieure du neurone. Le rôle physiologique de cette activité constitutive reste aujourd'hui inconnu.

Ces résultats dévoilent un nouveau concept : des RCPG non mutés peuvent être activés, dans des conditions d'hyperactivité cellulaire, par des protéines d'interaction intracellulaires, et ceci en l'absence de ligand extracellulaire. Bien que déclenché par des facteurs environnementaux, ce type d'activation dépend uniquement de l'état de la cellule elle-même et non plus uniquement des messages que lui envoient les cellules avoisinantes. Ce concept ouvre des perspectives nouvelles pour la concep-

tion de médicaments qui modifieraient l'association entre RCPG et protéines intracellulaires. $\nabla$

Receptor activation by intracellular protein: a novel concept

1. Bockaert J, Pin JP. Molecular tinkering of $G$ protein-coupled receptors: an evolutionary success. EMBO J 1999 ; $18: 1723-9$.

2. Morisset $S$, Rouleau $A$, Ligneau $X$, et al. High constitutive activity of native $\mathrm{H} 3$ receptors regulates histamine neurons in brain. Nature 2000 ; 408 : 860-4.

3. Ango F, Prezeau L, Muller T, et al. Agonist-independent activation of metabotropic glutamate receptors by the intracellular protein Homer.
Nature 2001 ; 411 : 962-5.

4. Xiao B, Tu JC, Worley PF. Homer: a link between neural activity and glutamate receptor function. Curr Opin Neurobiol 2000 ; 10 : 370-4.

5. Tu JC, Xiao B, Naisbitt $S$, et al. Coupling of mGluR/Homer and PSD-95 complexes by the Shank family of postsynaptic density proteins. Neuron 1999 ; 23 : 583-92.

6. Foa L, Rajan I, Haas K, et al. The scaffold protein, Homerlb/c, regulates axon pathfinding in the central nervous system in vivo. Nat Neurosci 20014 : 499-506.

7. Ango F, Pin JP, Tu JC, et al. Dendritic and axonal targeting of type 5 metabotropic glutamate receptor is regulated by homerl proteins and neuronal excitation. J Neurosci 2000 ; 20 : 8710 6.

8. Roche KW, Tu JC, Petralia RS, Xiao B, Wenthold RJ, Worley PF. Homer lb regulates the trafficking of group I metabotropic glutamate receptors. J Biol Chem 1999 ; 274 : 25953-7.

\title{
NOUVELLE
}

\section{Des cellules d'origine médullaire participent à la néoangiogenèse tumorale} Hèlène Gilgenkrantz

$>$ Au cours des premiers stades de la tumorigenèse, des cellules endothéliales sont recrutées et participent à la vascularisation de la tumeur. Une équipe newyorkaise tire parti d'un modèle connu de défaut d'angiogenèse, le modèle des souris $I d I^{+/-} I d 3^{-/-}$, pour démontrer que des cellules dérivées de la moelle osseuse participent à l'élaboration du réseau vasculaire tumoral.

Rappelons que les protéines HLH Id (dont il existe au moins 4 membres), en séquestrant des facteurs de transcription et en empêchent leur activité transcriptionnelle, se comportent comme des régulateurs dominants négatifs des facteurs de transcription bHLH, et sont requises pour des processus de différenciation aussi divers que la lymphopoïèse $B$, la neurogenèse ou l'angiogenèse.

On sait ainsi que tous les embryons de souris double knock-out $/ d I^{-/-} / d 3^{-/-}$ meurent à partir du $13,5^{\mathrm{e}}$ jour de développement d'hémorragies intracérébrales. Mais il est possible d'utiliser des souris $I d I^{+/-} / d 3^{-/-}$ayant une délétion homozygote du gène $I d 3$ et hétérozygote du gène $l d l$. Des expériences de xénogreffe de tumeurs chez ces souris ont révélé une réduction, voire une disparition totale, de la croissance tumorale et/ou une diminution du pouvoir méta-
Inserm U.129

ICGM, CHU Cochin,

24, rue du Faubourg Saint-Jacques, 75674 Paris Cedex 14, France.

statique de ces tumeurs. II en résulte une augmentation de la survie des animaux par rapport aux souris témoins non mutantes, qui meurent en quelques semaines [1]. II est intéressant de constater que l'inactivation d'un seul allèle Id est suffisante à la régression tumorale. Ce défaut de croissance tumorale est dû à une absence de vascularisation précoce de la tumeur [2]. Une des hypothèses pour expliquer cet effet angiogénique tumoral des protéines Idl et Id3 était leur contrôle de l'expression de l'intégrine $\alpha v \beta 3$ et de la métalloprotéinase MMP2. 
Afin de déterminer si les cellules impliquées dans l'angiogenèse étaient d'origine médullaire, les auteurs de l'article paru dans Nature Medicine [2] ont procédé à des transplantations de moelle issue soit d'animaux Rosa 26 (exprimant de façon constitutive la $\beta$-galactosidase), ou d'animaux $I d l^{+/-} / d 3^{-/-}$à des receveurs irradiés mutants $\left(I d l^{+/}\right.$ $\left.I d 3^{-/-}\right)$ou non. Après reconstitution médullaire, les animaux reçoivent une injection intradermique de cellules de carcinome ou de lymphome. Alors que les animaux sauvages ou mutants $I d l^{+/}$$1 d 3^{-/-}$transplantés avec une moelle normale meurent en quelques semaines, on observe un retard de croissance tumorale chez les animaux transplantés avec des cellules médullaires $1 d 3^{+/-} / d 3^{-/}$. Cette observation suggère que des cellules précurseurs de la moelle osseuse sont impliquées dans les phases précoces de l'angiogenèse tumorale. Dans les tumeurs développées chez les animaux transplantés avec la moelle Rosa 26 , plus de $90 \%$ des cellules des vaisseaux formés expriment la $\beta$-galactosidase, soulignant ainsi la contribution essentielle de ces cellules d'origine médullaire à l'élaboration du réseau vasculaire tumoral.

L'injection de VEGF (vascular endothelial growth factor) entraîne le passage, de la moelle osseuse vers la circulation, des progéniteurs hématopoïétiques et endothéliaux médullaires. La transplantation de cellules de souris Rosa26 ainsi «mobilisées » reconstitue, chez le receveur $I d 3^{+/-} \mid d 3^{-/-}$, non seulement l'ensemble des lignées hématopoïétiques, mais la vascularisation de la tumeur (lymphome ou carcinome) et donc la croissance tumorale. Dès le deuxième jour après l'implantation de cellules tumorales chez ces receveurs, la majorité des néovaisseaux formés expriment la $\beta$-galactosidase dans les deux types tumoraux étudiés. Les cellules endothéliales $\mathrm{LaCZ}^{+}$sont également associées à des cellules mononucléées

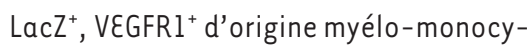
taire $\left(\mathrm{MOMA}^{+}, \mathrm{CDllb} \mathrm{b}^{+}\right)$au sein des tumeurs. Deux semaines plus tard, la proportion de cellules provenant $d u$ donneur est plus importante après implantation de cellules de lymphome (90\%) qu'après implantation de cellules de carcinome (50\%), suggérant que l'implication de ces précurseurs médullaires dans le maintien de ces néovaisseaux varie avec la nature de la tumeur.

Cette restauration d'une angiogenèse tumorale à partir de cellules médullaires mobilisées rappelle les observations récentes faites dans l'infarctus expérimental chez la souris: des progéniteurs médullaires mobilisés par une autre cytokine, le G-CSF, étaient capables de reconstituer une vascularisation dans une zone nécrotique du cœur induite par la ligature de l'artère coronaire. Il est donc certain que des précurseurs endothéliaux circulent, et qu'ils peuvent contribuer à la vascularisation tumorale aussi bien que normale.

L'élévation du VEGF plasmatique chez les souris sauvages induit une mobilisation de précurseurs endothéliaux exprimant le récepteur VEGFR2 et n'exprimant pas le marqueur de la lignée myélomonocytaire CDllb. L'inoculation de cellules tumorales de lymphome induit également la mobilisation de cellules VEGFR2+ circulantes. À l'inverse, chez les souris mutantes, dans les deux schémas expérimentaux (induction de
VEGF circulant ou injection de cellules tumorales), ce recrutement n'a pas lieu. Afin de déterminer l'implication de la mobilisation des précurseurs médullaires VEGFR2 ${ }^{+}$et/ou des précurseurs VEGFR1 ${ }^{+}$ dans l'angiogenèse tumorale, des souris sauvages ayant reçu une xénogreffe tumorale ont été traitées par des anticorps dirigés contre le récepteur $\mathrm{du}$ VEGFR1, du VEGFR2 ou contre les deux types de récepteurs. Le ciblage de l'un ou de l'autre des récepteurs isolément ne bloque que partiellement la croissance tumorale, alors que l'inhibition des deux récepteurs suffit pour bloquer totalement le développement de la tumeur.

Ces résultats ne sont donc pas seulement importants pour la compréhension des mécanismes qui président à la croissance tumorale précoce, mais ils désignent également une double cible thérapeutique anti-tumorale: les précurseurs endothéliaux exprimant le récepteur VEGFR2 et certaines cellules provenant de la moelle et exprimant le récepteur VEGFRI. $\diamond$

Endothelial cells organizing vessels within tumors are derived from the bone marrow

1. Lyden D, Young AZ, Zagzag D, et al. Id $\mathrm{l}$ and $\mathrm{Id} 3$ are required for neurogenesis, angiogenesis and vascularization of tumour xenografts. Nature $1999 ; 401: 670-7$

2. Lyden D, Hattori K, Dias $S$, et al. Impaired recruitment of bone-marrow-derived endothelial and hematopoietic precursor cells blocks tumor angiogenesis and growth. Nat Med $2001 ; 7: 1194-01$ 